\title{
IoT Based Waste Tracking and Collection
}

\section{Akshay Patil, Vitthal Sawant, Sarvesh Kothawade, Prof. Sonal Chaudhari}

Department of Computer Engineering, Datta Meghe College of Engineering, Airoli, Navi Mumbai, Maharashtra, India

\section{ABSTRACT}

\section{Article Info}

Volume 7, Issue 3

Page Number: 76-84

\section{Publication Issue :}

May-June-2021

\section{Article History}

Accepted : 01 May 2021

Published : 07 May 2021
Waste collection and resource management on time has been always a challenge rather efficient collection of waste by corporation. This project aims to achieve this goal with help of Internet of Things and mobile application and intend to retrieve data from citizens to help us efficiently manage collection triggers of collection vehicle and resources according to need, so that productivity of waste collection increases. Data suggest that in urban as well as rural area management struggles with the efficiency and resource planning as there exist few or no management system which will fit all scenarios. As we know data is new age oil. We intend to generate data that can be useful in various aspects ranging from analysis of public health to resource planning and as proof of concept we will demonstrate how we can efficiently utilize resources i.e vehicle.

Keywords : Cloud Computing, Encryption, Homomorphic Encryption, Fully Homomorphic

\section{INTRODUCTION}

We are living in an age where tasks and systems are fusing together with the power of IOT and software systems to have a more efficient system of working and to execute jobs quickly! With all the power at our finger tips this is what we have come up with. The Internet of Things (IoT) shall be able to incorporate data collection and monitoring in different systems, while providing data from millions of people to use and capitalize. Building a general architecture for the IoT is hence a very complex task, mainly because of the extremely large variety of devices, link layer technologies, and services that may be involved in such a system. One of the main concerns with our environment has been solid waste management which impacts the health and environment of our society. The tracking, monitoring, collection and resource management of waste is one of the primary problems of the present era. The traditional way of periodic collection the wastes is a cumbersome process and inefficient, more human effort, time and cost which can easily be avoided with this project based on data collection. A System in, which waste data is tracked and analyzed.

\section{Problem Definition}

Nowadays, there are tons of flats and apartments which have been built in the rapid urbanization area. This is due to high housing demands which have been drastically raised as a result of migration from villages to cities to find work. In order to accommodate the growing population in the urban area, the government has also constructed more apartment complexes. There are several issues faced by the residents of the flats. One of them is disposal 
of solid waste. Unlike private houses, the residents of all the apartments use a common dustbin, which tends to fill up very quickly. This overflowing of garbage is a sanitary issue which might cause diseases like cholera and dengue. Moreover it is a waste of fuel to travel around a complex or an area to find that some of the garbage is filled and some are not. Also, on rare days, problems might arise that there is so much garbage that the truck doesn't have enough capacity hence showing poor resource management. This happens due to inability to know which, how much, where, when the resource is required all this questions can be answered with the help of data. On the contrary hand corporation struggle with management and decision making to overcome this problem software system is useful. On top of that costly hardware infrastructure to implement similar solution could be waste of money.

\section{Aim and objectives}

- Measure weight of waste by IoT sensors (Generate Data).

- $\quad$ Sending measured weight via Bluetooth to client app

- Data tracking and uploading it to cloud server

- Analysis based on data collected on admin app

- Trigger waste collection protocol for vehicle.

- Plan resource according to need.

- Create revenue generation echo system by engagement of citizens.

\section{Literature Review}

\section{[1] IoT-Based Smart Garbage System for Efficient} Food Waste Management.

In this literature we understood the working of mass software and hardware infrastructure but the application of this research paper was to target public waste on the cost of very complex and expensive infrastructure. From this literature we got knowledge on various approach for solution and scope for improvement

\section{[2] IoT Based Smart Garbage and Waste Collection Bin.}

In this literature we understood the hardware is effective as compare to above literature ${ }^{[1]}$ but not overall approach to the solution as its aim is to manage public dustbins using hardware sensors that can be reduced in infrastructure and cost.

\section{[3] The Working Principle Of An Arduino.}

This literature gives us knowledge of working principles of arduino. Which help us understand basic protocol and handling of Input output pins. As well as give us knowledge about IDE to upload custom code.

By reviewing papers we find out many of solution revolves around inefficient and high cost hardware infrastructure. With the intentions to make hardware reach to every user we must focus on reducing cost of hardware infrastructure other paper using extra module to perform same operation ex. Sonar based sensors to recognize whether the bin is full or not. To transfer data between servers they use Wi-Fi modules that are dependent on internet routers in home and subjected to availability hence increasing dependency and cost of hardware infrastructure. On software side we are using client app to transfer collected data to server hence reducing dependency on other hardware than mobile.

\section{Requirement Analysis}

\section{Software Requirements}

We will need the following software to accomplish our project. 
1. Editor for writing code eg. Vscode, sublimetext

2. Arduino IDE for writing microcontroller code

3. Web Browser for running testing and preview the functionality e.g. Google Chrome

4. Node.js, express, mongodb, react for admin panel development

5. MIT app inventor a block coding tool for mobile app development only for (prototype)

\section{Hardware Requirements}

We will need the following hardware to accomplish our project.

\section{Arduino Uno}

2. Load cell

3. Scale sensor cum signal amplifier e.g (HX 711)

4. Bluetooth Module i.e (HC-06)

5. Strain gauge for placing load(platform)

6. Usb cable for connecting arduino to computer and write data

7. Wooden slits as platform to rest weight on(prototype only)

\section{Methodology}

To tackle unknown question set i.e when on average time citizen generates waste? Where demographically currently waste is being generated? Where should according to priority resource should be send to collect waste? Which demographic has assigned how much resource? Is that resource sufficient or excess for that region? Is health of people living in area where waste is generated frequently affected compare to people living in area where waste generation rate is low etc. all such questions and problem can be solved by the data.

To generate data with the help of technology system need affordable hardware and software system to monitor and analyse for administration and citizen.
With generation of mature data we will be able to decide which demographic is currently filled with waste hence triggering waste collection protocol which in sense will effectively increase productivity and efficiency.

Now talking about software, this project provide software solution or system for both user and administration.

So the "tracking" part in the title of project is subjected to Generation of data using hardware, transaction of data using software and the "collection" part of title of project is subjected to analysis on data perform by software, triggering waste collection protocol and resource management is also subjected to analysis on data perform by software. How to capitalize this system and make citizen to engage in the process? Well, implement system that offers credit points to citizens on successfully throwing waste in home bin. Which on accumulation can be redeem to purchase from in-app offers and to redeem coupon code. But the entity's offering this offers will pay the system commission on the sale of their product and offer utilization. Creating an ecosystem of advertisement and sale's module hence, generating revenue to operational authority.

To tackle hardware cost problem we came up with idea, now a days citizens have more powerful hardware that is mobile phone so system don't need separate hardware to be implemented in public domain hence reducing access cost. On top of that system will offer minimalistic hardware to generate data that is affordable by citizen rather cost of hardware is recoverable over period of the time which makes hardware technically FREE! 


\section{System Architecture}

\section{Hardware}

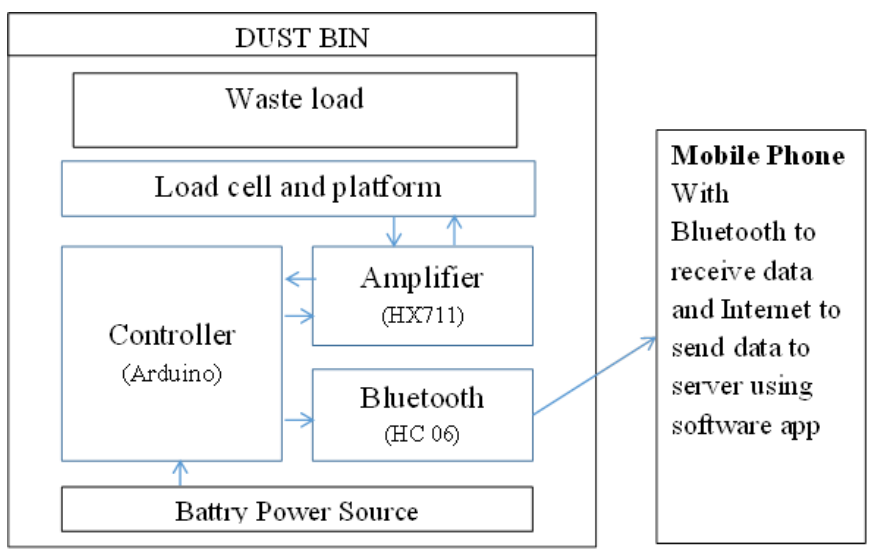

Figure 1. Single Hardware Node (SHN) or smart bin

Single hardware node (SHN) is complete set of hardware that generates data and send to mobile phone. Thus more than one SHN in close proximity is set of demographic cluster. More the number of SHN in close proximity more the dense cluster is. a number of set in cluster is subject to acceptance by citizens. According to demographic of city we can observe multiple clusters that could be form.

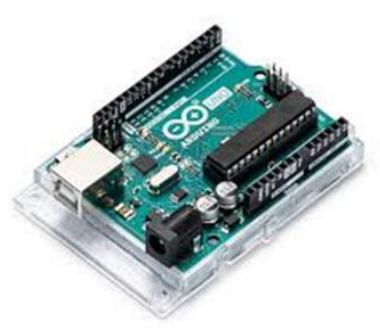

Figure 2. arduino uno microcontroller [6]

The hardware part of the system mainly includes Arduino board UNO and development environments. Arduino is an open-source development of I/O interface control panel. There is a similar java, C language development environment, so that users can use the Arduino language and other software to make interactive works. Many switches or sensors, LED, stepper motors, etc. There are many amazing interactive work can be developed through the Arduino. ${ }^{[3]}$

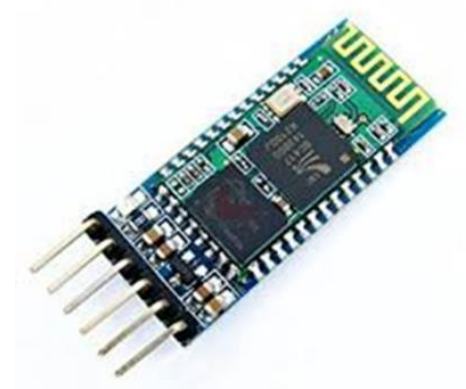

Figure 3. Bluetooth Module HS-06 [7]

The Bluetooth Module HC-06 is a short-distance communication tool, connect handheld device and connect Arduino. Its power requirements are $3-5 \mathrm{~V}$ and microcontroller can be connected directly. It has Bluetooth data transmission capabilities. Bluetooth support remote IO control mode. In this paper, we will use the Bluetooth module HC-06 to do the communication between Arduino and smart phone. ${ }^{[4]}$

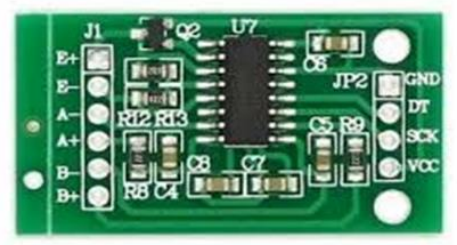

Figure 4. HX-711 signal amplifier or scalar [8]

HX711 is a specially designed 24-bit A/D converter chip for high-precision electronic scale. Compared with the same type of other chip that integrates including power supply, peripheral circuitry on-chip clock oscillator and other needed the same type of chip. It has high integration, fast response, antiinterference and other advantages. Reducing the overall cost of electronic scale and improve the overall performance and reliability. In this paper we will use the scale's sensor HX711 as A/D converter for load cell. ${ }^{[4]}$ 


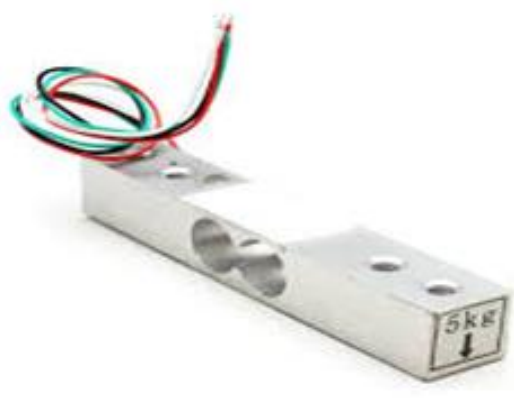

Figure 5. load cell [9]

In bar strain gauge load cells, the cell is set up in a " $Z$ " formations so that torque is applied to the bar and the four strain gauges on the cell will measure the bending distortion, two measuring compression and two tension. When these four strain gauges are set up in a Wheatstone bridge formation, it is easy to accurately measure the small changes in resistance from the strain gauges. A strain gauge is a device that measures electrical resistance changes in response to, and proportional of, strain (or pressure or force or whatever you so desire to call it) applied to the device. The most common strain gauge is made up of very fine wire, or foil, set up in a grid pattern in such a way that there is a linear change in electrical resistance when strain is applied in one specific direction, most commonly found with a base resistance of $120 \Omega, 350 \Omega$, and $1,000 \Omega$. Each strain gauge has a different sensitivity to strain, which is expressed quantitatively as the gauge factor (GF). The gauge factor is defined as the ratio of fractional change in electrical resistance to the fractional change in length (strain). (The gauge factor for metallic strain gauges is typically around 2 . ${ }^{[4]}$

\section{Software}

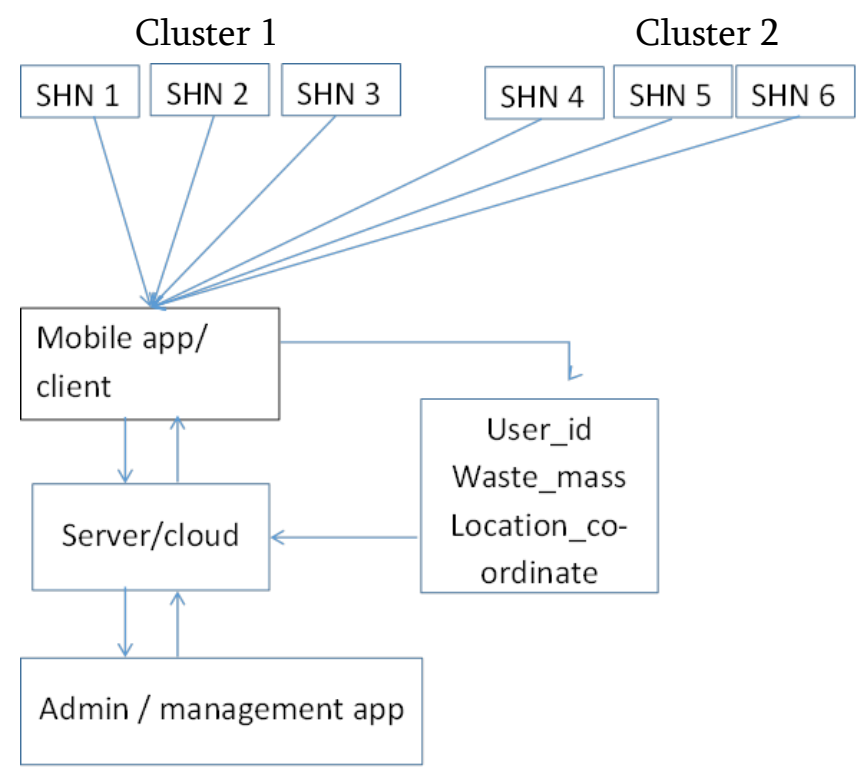

Figure 6. Software Layers

Software architecture is responsible for processing and analysis of data ${ }^{[1]}$. Data is collected from many SHN. This data collects weight of waste and GPS coordinates of node or smart bin than sends this data from users phone to server where this data is processed and according to co-ordinates location cluster is formed. The data can be analyze to resource management and waste collection

\section{Block Diagram}

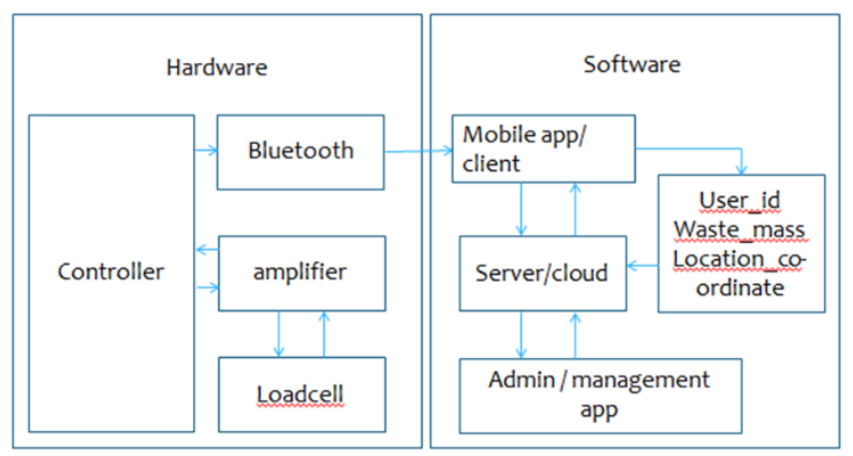

Figure 7. Block Diagram 


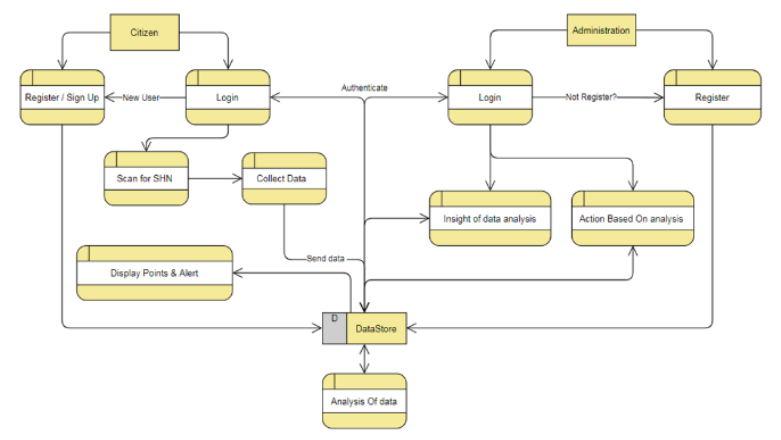

Figure 8. Level 1 Data Flow Diagram[5]

\section{Design Implementation}

\section{Hardware Implementation}

The process of hardware design is as follows. Use screws to fix the two surfaces of the scale and strain beam. ${ }^{[4]}$ To making both sides is in limbo, under the action of gravity. The deformation of strain beam will cause the strain gauge at the sealant to let resistance changes and to generate a signal ${ }^{[4]}$. The strain gauge has direction while in the opposite direction, the weighing value is negative. In this experiment, the range of the strain beam is $5 \mathrm{~kg}$ to $10 \mathrm{~kg}$. The scale sensor module has VCC, GND, DOUT, SCK respectively to connect to Arduino's power supply 3.3V, GND, A1, A0. in addition, VCC, GND, TXD, RXD of the Bluetooth Module HC-06 respectively connected to Arduino's 3.3V, GND, RX, TX. The Arduino is connected via USB to link to the computer. Then, the connection of the Bluetooth electronic scale's hardware is completed ${ }^{[3][4]}$.

When user puts waste in bin on platform the load is calculated with the help of train beam (load cell) and signal is scaled with the help of HX711. ${ }^{[4]}$ This data then transmitted via Bluetooth module on controller to receiver (mobile phone app). Microcontroller must be code in order to perform this operation. Microcontroller is able to make different module communicate with each other. Power required for hardware assembly can be provided by battery cell or by computer ports for testing purpose.

\section{Software Implementation}

Then there starts software part in which mobile app sends this weight of waste, gps co-ordinates, user id to server or cloud. After receiving data from SHN server constantly analyses the data and admin is able to see all stat in real-time. Main functionality of software is to collect and analyse data and according to analysis trigger waste collection protocol. The data is also able to analyse and suggest planning resource accordingly. E.g. consider two areas of metro city with respective corporation. Now there exist mature amount of SHN to collect data in both cities when data is received by server it is able to analyse which city has what rate of waste collection. In case one, city 1 generates 10 ton waste/day and has 8 waste collection vehicles. In case two, city 2 generates 5 ton waste/ day and has 10 waste collection vehicles. By this resource and data stat we are able to analyse and calculate ratio of vehicle need for city $1>$ city 2 hence we can transfer 3-4 vehicle from city 2 to city 1 hence our software is able to manage resource efficiently. Now speaking about implementation we can create web interface for administration and mobile app for client side which simply means app for citizens. Then user will install on their mobile devices and register on the same. Client app will use Bluetooth and gps module of mobile for data collection. Admin app is able to show graphical insights of data being generated and able to monitor rather trigger protocols. System will also have gamification client app in which user will be assign points according to their engagements and revenue generation module. Insights on admin app can be able to sort data according to demographic, cluster, unique id and also able to sort by date hence providing various means for analysis. Client app will be able to notify about waste collection trigger. Hence alerting users of planned pickups. The stat generated from system can also be counter examine for research on effects of health on cluster that 
generates more waste than other and can be useful to take decisions in health domain accordingly.

\section{Working Prototype and Output}

\section{SHN (Single Hardware Node)}

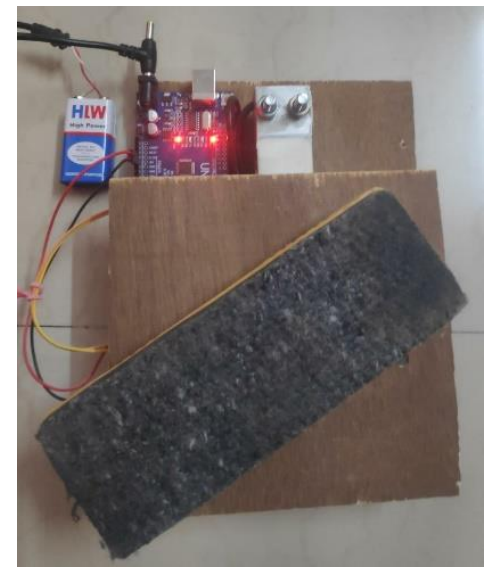

Figure 9. SHN with load on top

Working prototype of SHN is successfully able to read and transmit data.

\section{Client Mobile App}
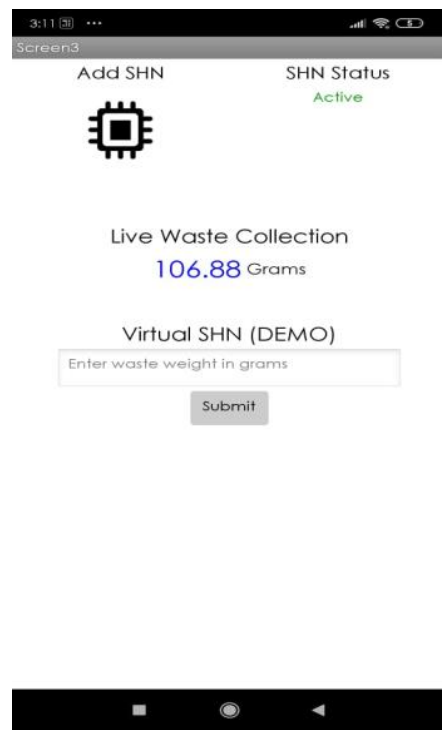

Figure 10. App screen showing weight.

Data transmitted via Bluetooth is being shown in client mobile app and then send to server realtime.

\section{Admin Panel}

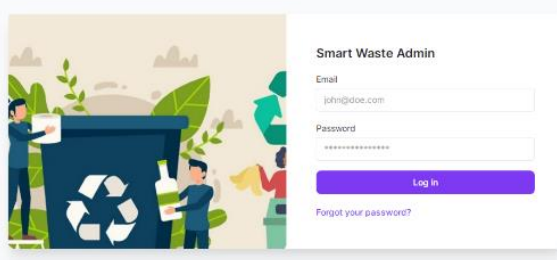

Figure 11. Admin Login screen
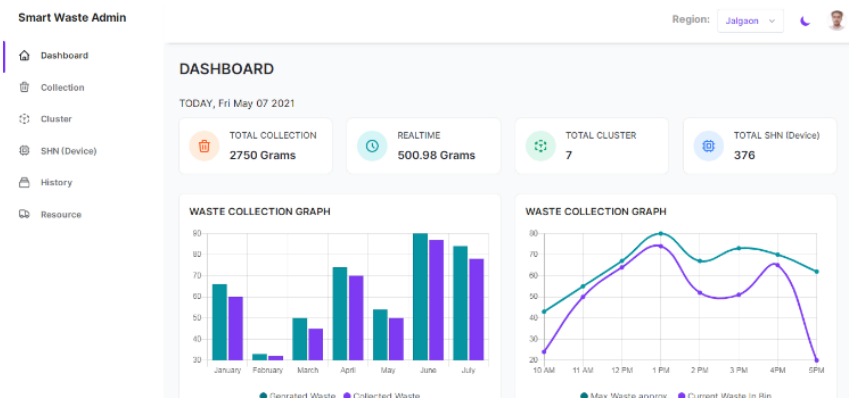

Figure 12. Admin Dashboard showing weight data by region

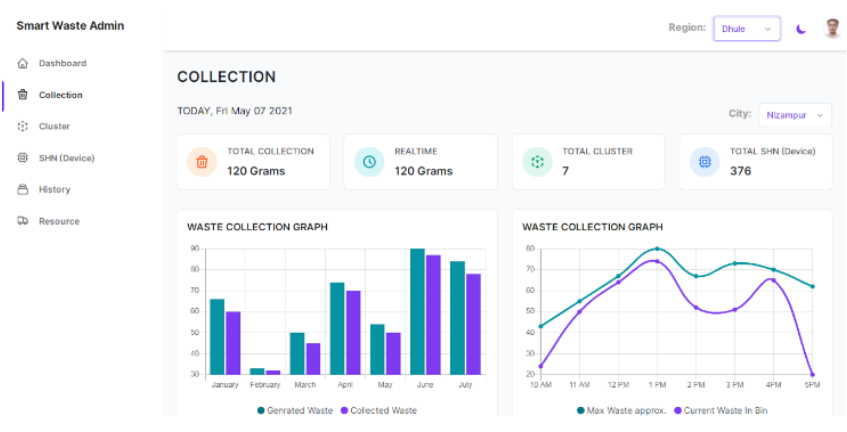

Figure 13 . Waste collection detail by city

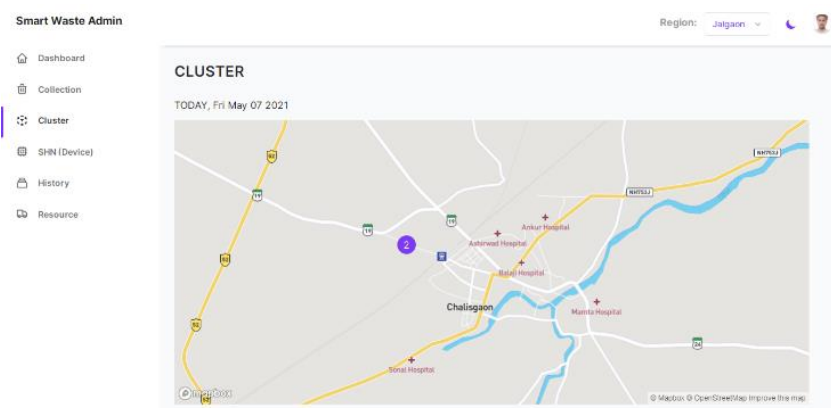

Figure 14. Cluster map showing formed cluster

Admin panel receives data from server in real-time on that basis admin is able to choose region and the 
data of that region is being shown on dashboard. In collection section admin can choose to see collection by city of selected region. Cluster here showing cluster in "jalgaon" region and the cluster of two user is formed in "chalisgaon" city hence we were able to successfully develop working prototype of complete proposed system architecture.

\section{CONCLUSION}

Project is an efficient waste monitoring system that can be used to monitor the level of waste and able to collect data for analysis. This data can be further used to plan garbage collection trips, ultimately increasing efficiency, also it can be able to plan and manage resources according to need, creating revenue business modal though ad and offer promotion.

\section{FUTURE SCOPE}

To extend this project in public domain as phase one will create enough data rather mature data to analyze on. In phase two this project intend to create system independent of SHN by creating system that will allow user to just throw trash in bag printed with unique $\mathrm{QR}$ code and is made of corn starch which is decomposable and organic. user can purchase this bag at very cheap price on app or shop vendor and register purchased set of bag using $Q R$ code to system directory via app. so that when they throw trash system can compare $\mathrm{QR}$ code with database and would know that, to whom this bags belongs. On the solid waste management site state of art separator will classify waste using image processing. Users just have to fill trash in bag with unique code and throw in any bin. If user is outside (not at home or society) and finds trash on road he/she can pick up trash and put in smart bin installed at public places in process user have to scan code given on trash can using mobile app and place trash in the bin. Bin is equipped with weight sensor that will measure weight of trash just thrown by user and will reflect points in users profile for throwing trash.

\section{ACKNOWLEDGEMENT}

Motivation and guidance are the keys towards success. i would like to extend my thanks to all the sources of motivation.

We would like to grab this opportunity to thank Dr. S. D. Sawarkar, principal for encouragement and support he has given for our project.

we express our deep gratitude to Dr. A. P. Pande, head of the department who has been the constant driving force behind the completion of this project.

We wish to express our heartfelt appreciation and deep sense of gratitude to my project guide Prof. Sonal Chaudhari for her encouragement, invaluable support, timely help, lucid suggestions and excellent guidance which helped us to understand and achieve the project goal. her concrete directions and critical views have greatly helped us in successful completion of this work.

We extend our sincere appreciation to all professors for their valuable inside and tip during the designing of the project. Their contributions have been valuable in so many ways that we find it difficult to acknowledge of them individually.

We are also thankful to all those who helped us directly or indirectly in completion of this work.

\section{REFERENCES}

[1]. IoT-Based Smart Garbage System for Efficient Food Waste Management. By Insung Hong, Sunghoi Park, Beomseok Lee, Jaekeun Lee, 
Daebeom Jeong, and Sehyun Park. Aug 2014

Source:https://www.researchgate.net/publication /266252204_IoT-

Based_Smart_Garbage_System_for_Efficient_Fo od_Waste_Management

[2]. IoT Based Smart Garbage and Waste Collection Bin. By S.S.Navghane, M.S.Killedar, Dr.V.M.Rohokale. May 2016 Source:http://ijarece.org/wpcontent/uploads/2016/06/IJARECE-VOL-5ISSUE-5-1576-1578.pdf

[3]. The Working Principle Of An Arduino. By Yusuf Abdullahi Badamasi,Nigerian Turkish Nile University. Dec 2014 Source:https://ieeexplore.ieee.org/document/699 7578

[4]. Using Arduino to Develop a Bluetooth Electronic Scale for Water Intake. By Bin Dai, Rung-Ching Chen, Wei-Bin Yang Source:https://ieeexplore.ieee.org/document/754 5302

[5]. Tool used for diagram drawings https://online.visual-paradigm.com/

[6]. Arduino:

https://www.jameco.com/Jameco/Products/MPi mages/2152366A.jpg

[7]. Bluetooth https://www.instructables.com/Howto-Set-AT-Command-Mode-for-HC-05Bluetooth-Mod/

[8]. Signal amplifier https://www.instructables.com/Build-a-Build-aDigital-Weight-Scale-Force-Guage/

[9]. load cell https://www.lazada.com.my/products/load-cellstraight-bar-0-5kg-weight-sensor-for-arduinoiot-i445496075.html

\section{Cite this article as :}

Akshay Patil, Vitthal Sawant, Sarvesh Kothawade, Prof. Sonal Chaudhari, "IoT Based Waste Tracking and Collection", International Journal of Scientific Research in Computer Science, Engineering and Information Technology (IJSRCSEIT), ISSN : 24563307, Volume 7, Issue 3, pp.76-84, May-June-2021. Available at doi : https://doi.org/10.32628/CSEIT217319 Journal URL : https://ijsrcseit.com/CSEIT217319 